\title{
A Comparison of the Effects of Different Perspectives in Terms of Reappraisal Subtypes on Affect: Positive Reappraisal and Putting into Perspective
}

\author{
Megumi Oikawa1 ${ }^{1}$, Akio Nakano ${ }^{2}$, Rie Tabuchi ${ }^{3}$ \\ ${ }^{1}$ Faculty of Education, Tokyo Gakugei University, Tokyo, Japan \\ ${ }^{2}$ A Graduate of Graduate School of Education, Tokyo Gakugei University, Tokyo, Japan \\ ${ }^{3}$ The United Graduate School of Education, Tokyo Gakugei University, Tokyo, Japan \\ Email: oikwmgm@u-gakugei.ac.jp
}

How to cite this paper: Oikawa, M., Nakano, A., \& Tabuchi, R. (2017). A Comparison of the Effects of Different Perspectives in Terms of Reappraisal Subtypes on Affect: Positive Reappraisal and Putting into Perspective. Psychology, 8, 2047-2057.

https://doi.org/10.4236/psych.2017.812131

Received: August 28, 2017

Accepted: October 23, 2017

Published: October 26, 2017

Copyright (ङ 2017 by authors and Scientific Research Publishing Inc. This work is licensed under the Creative Commons Attribution International License (CC BY 4.0).

http://creativecommons.org/licenses/by/4.0/

(c) (i) Open Access

\begin{abstract}
Reappraisal has attracted attention as one of the effective emotion regulation strategies. In recent years, it has been suggested that reappraisal has some subtypes. However, the effects of the subtypes on affect have not yet been investigated in detail. In the current study, we focused on "positive reappraisal" and "putting into perspective" as reappraisal subtypes. The purpose of this study was to experimentally investigate the effects of the two subtypes on affect. Participants were 107 undergraduate and graduate students $(57$ males and 50 females) from a Japanese university. They were randomly assigned to one of two conditions (positive reappraisal or putting into perspective). They completed a questionnaire to evaluate three types of affect at baseline, after a stress induction task, and after a reappraisal task. A $t$-test was conducted of the change score of each type of affect during the reappraisal task. The positive reappraisal group showed more of an increase in active positive affect and more of a decrease in depression and anxiety compared with the putting into perspective group. The result for non-active positive affect was not significant. In conclusion, positive reappraisal was a more effective strategy for emotion regulation than putting into perspective. The findings also indicate that putting into perspective was as effective for increasing non-active positive affect as positive reappraisal.
\end{abstract}

\section{Keywords}

Reappraisal, Subtype, Positive Reappraisal, Putting into Perspective 


\section{Introduction}

Emotion regulation plays a very important role in maintaining and improving mental health. When we experience an unpleasant event and feel negative emotion, thinking about the event might be important for problem solving and emotion regulation. However, merely thinking about the negative event or emotion repeatedly is likely to lead to the inhibition of emotion recovery and problem solving (Nolen-Hoeksema, Wisco, \& Lyubomirsky, 2008). In recent years, reappraisal has attracted attention as one of the effective emotion regulation strategies. Reappraisal involves cognitively transforming the situation in order to alter its emotional impact (Gross, 1998a). The effects of reappraisal have been compared with suppression, which involves inhibiting emotion-expressive behavior, and many studies have suggested that reappraisal is a more adaptive strategy than suppression (e.g., Gross, 1998b; Hofmann, Heering, Sawyer, \& Asnaani, 2009).

Recently, it has been suggested that reappraisal has some subtypes, such as focusing on the positive sides of the event or seeing the event from a comparative perspective, which are ways of reevaluating a negative situation. McRae, Ciesielski, \& Gross (2012) have identified eight categories of reappraisal subtypes focusing on goals and tactics (e.g., reducing negative emotions or enhancing positive emotions). The results of a meta-analysis of reappraisal experiments (Webb, Miles, \& Sheeran, 2012) indicate that reappraisal has three types that are based on the target of the reappraisal, and all three strategies are effective, although a mixture of the three strategies is the most effective. In addition to the above research studies, the Cognitive Emotion Regulation Questionnaire (CERQ) (Garnefski, Kraaij, \& Spinhoven, 2001) is a very useful scale for assessing various cognitive strategies, including the subtypes of reappraisal (Sakakibara, 2014). Although the classification of the subtypes in previous research was not always equivalent, it has been demonstrated that reappraisal has some subtypes and that the effects of reappraisal on affect differ according to the subtypes. Recent studies have started to focus on the effects of the subtypes on affect. However, the effects of the subtypes of the strategies on affect have not been investigated in detail. Investigating the effects of the subtypes is very important from the point of view of using a strategy in a more adaptive way.

The CERQ assesses the following nine cognitive strategies: self-blame, blaming others, acceptance, refocus on planning, positive refocusing, rumination or focus on thought, positive reappraisal, putting into perspective, and catastrophizing. In this study, we focused on positive reappraisal and putting into perspective from the CERQ as the reappraisal subtypes, because the two strategies might especially relate to changing the appraisal of the situation. The former refers to thoughts that attach a positive meaning to the event in terms of personal growth, while the latter refers to thoughts that play down the seriousness of the event or emphasize its relativity when compared to other events (Garnefski et al., 2001). The two strategies have similarities in that both change one's view of 
events and they are theoretically included in the group of more adaptive strategies (Garnefski et al., 2001). However, while positive reappraisal refers to an active perspective from a positive side (e.g., "I could grow through experiencing this event"), putting into perspective refers to a lower comparative perspective (e.g., "It was not the worst result that could have happened"). Therefore, these two strategies are contrasting in terms of their difference in perspective.

Previous research studies have suggested that positive reappraisal is an effective strategy to reduce negative emotion. Garnefski \& Kraaij (2007) showed that only positive reappraisal had prospective relationships with depressive and anxiety symptoms, and that putting into perspective had no relationship with either symptom. Moreover, in other research, putting into perspective has been shown to be related to higher levels of depressive symptoms (Schroevers, Kraaij, \& Garnefski, 2007). On the other hand, there are studies that show that both strategies have a negative relationship with negative symptoms (e.g., Jermann, Van der Linden, d'Acremont, \& Zermatten, 2006). It is also suggested that positive reappraisal is effective for enhancing positive emotion. In an experiment that examined the effect of cognitive strategies on positive and negative affect after a stress induction task, a positive reappraisal group showed a significant increase in their positive affect and a decrease in their negative affect compared with other cognitive strategy groups (i.e., rumination, acceptance, and distancing) (Rood, Roelofs, Bögels, \& Arntz, 2012).

Although the effects of positive reappraisal and putting into perspective on affect have not always been consistent, many studies have suggested that positive reappraisal is an effective strategy for reducing negative emotion and enhancing positive emotion. Sakakibara \& Kitahara (2016) investigated the relationships between the CERQ and depression and anxiety through a meta-analysis, and they found that positive reappraisal and putting into perspective had significantly negative correlations with both variables, and that the correlations between putting into perspective with both variables were weak relatively. Therefore, from a comprehensive viewpoint, we anticipated that the change in affect would be greater when using positive reappraisal than when using putting into perspective. However, no study has compared the two strategies experimentally because most of the research studies have examined the effects of the two strategies through self-report using the CERQ. Thus, in this research, we experimentally investigated the effects of the two strategies on negative and positive affect. We assessed depression and anxiety as negative affect, and we assessed different types of affect as positive affect, which included active and non-active positive affect (i.e., energetic and calm respectively), in order to investigate the different effects of the two strategies in detail. We hypothesized that an increase in positive affect and decrease in depression and anxiety would be greater in a positive reappraisal group than in a putting into perspective group. Because most previous studies have not investigated the effects of the strategies on non-active positive affect, we could not hypothesize about each type of positive affect sepa- 
rately. In order to investigate the hypotheses, we conducted an experiment to compare the two strategies in terms of the change in each type of affect before and after completing a reappraisal task.

\section{Methods}

\subsection{Participants}

The participants were 107 undergraduate and graduate students (57 males and 50 females) from a Japanese university. The mean age was 22.05 years $(S D=$ 1.77). Participants were recruited widely through announcements in university classes or announcements through student email lists. We asked those who offered to cooperate to participate in this study.

\subsection{Affect Assessment}

Both groups completed a short version of the Multiple Mood Scale (Terasaki, Kishimoto, \& Koga, 1992) to measure the following three types of affect: active positive affect, non-active positive affect, and depression and anxiety. This scale measures the multifaceted subjective emotional state. Each type of affect was assessed by five items that were rated on a four-point Likert-type scale (i.e., $1=n o t$ at all to $4=$ feel clearly). Active positive affect was assessed by such items as "energetic", while non-active positive affect was assessed by such items as "calm". Depression and anxiety were assessed by such items as "worried". Depression and anxiety were not measured as a symptom but as a negative emotional state.

\subsection{The Stress Induction Task}

Participants were instructed to recall a current or recent stressful experience for the stress induction task (cf. Rood et al., 2012). They imagined the event as a movie, and remembered the feelings and sensations related to the event until they could see the event vividly and they felt like they were experiencing the event again. Then, they stopped imagining the most stressful moment of the event. The participants continued the task for 3 minutes.

\subsection{The Reappraisal Task}

The instruction for the two reappraisal subtypes was based on items from the Japanese version of the CERQ (Sakakibara, 2015) and the instruction of Rood et al. (2012). Participants were instructed to think about their experience again as instructed.

The positive reappraisal group was instructed to think about the positive sides of the event and to examine the learning from the event and how the event enabled them to grow. The putting into perspective group was instructed to think that the event could have become much worse, and that other people go though much worse experiences. Each group continued the task for 5 minutes.

In order to confirm that the participants were engaged in the assigned task, 
three items corresponding to each strategy were used (cf. Rood et al., 2012; Sakakibara, 2015). An example of an item corresponding to positive reappraisal is "I thought about the positive things that I could get out of the event/situation." An example item corresponding to putting into perspective is "I thought that it could have been much worse." After finishing the reappraisal task, the participants rated these six items on a six-point Likert-type scale (i.e., $1=$ not at all to 6 = very applicable).

\subsection{Procedure}

The experimenter explained the outline of the study to the participants, and asked who was willing to participate. After obtaining informed consent, the experiment was conducted individually in university classrooms. The experiment took approximately 20 minutes to complete. The participants were randomly assigned to one of two conditions: the positive reappraisal group $(n=54)$ and the putting into perspective group $(n=53)$.

Both groups completed a questionnaire to measure the baseline affect (Time 1). Then, they were instructed to recall a recent negative event and their affect while experiencing that event, and affect was assessed (Time 2). Next, they participated in a different reappraisal task for each condition. After the reappraisal task, they rated items that assessed the manipulation check and affect (Time 3). Finally, a distraction task was introduced to recover the participants' negative affect to the same level as at the baseline for ethical reasons. The task was a logic puzzle in which dots were connected in turn to complete a picture. After finishing the distraction task, depression and anxiety were measured. This study was carried out with the approval of the ethics committee of the affiliated university.

\subsection{Statistical Analyses}

The planned analyses included a reliability analysis and the calculation of the basic statistics for each scale. We conducted a 2 (time: the baseline and after the stress induction task) $\times 2$ (group: the positive reappraisal group and the putting into perspective group) mixed-model two-way analysis of variance for the manipulation check of the stress induction task. T-tests were conducted for a manipulation check of the reappraisal task and for the change in each type of affect before and after the reappraisal task. Finally, the recovery of negative affect was investigated with a 2 (time: the baseline and after the distraction task) $\times 2$ (group: the positive reappraisal group and the putting into perspective group) mixed-model two-way analysis of variance. All analyses were conducted with SPSS version 21.0 (IBM Corp., Armonk, NY, USA).

\section{Results}

\subsection{Manipulation Check}

We investigated the effects of the stress induction task on each type of affect with a mixed-model two-way analysis of variance. For active positive affect, the inte- 
raction and the main effect of group were not significant $(F(1,105)=0.84, F(1$, $105)=0.12)$. The main effect of time was significant $(F(1,105)=116.92, p<$ $0.001)$. For non-active positive affect, the interaction and the main effect of group were not significant $(F(1,105)=0.30, F(1,105)=0.15)$. The main effect of time was significant $(F(1,105)=101.75, p<0.001)$. For depression and anxiety, the interaction and the main effect of group were not significant $(F(1,105)=$ $0.37, F(1,105)=1.20)$. The main effect of time was significant $(F(1,105)=66.72$, $p<0.001)$. The participants showed a decrease in the two types of positive affect and an increase in depression and anxiety through the stress induction task.

We conducted a $t$-test to confirm that the participants were engaged in the assigned task. The reliability of the two scales corresponding to each condition was high for positive reappraisal $(\alpha=0.82)$. Although the putting into perspective scale showed a slightly low score $(\alpha=0.63)$ compared with the other scale, we used these two scales because the score was not problematic.

The mean score of the positive reappraisal was $13.28(S D=2.84)$ for the positive reappraisal group and $8.45(S D=3.73)$ for the putting into perspective group $(t(97.17)=7.51, p<0.001)$. The mean score of putting into perspective was $6.80(S D=2.45)$ for the positive reappraisal group and $12.13(S D=2.72)$ for the putting into perspective group $(t(105)=10.67, p<0.001)$. These results show that the participants were engaged in each task.

\subsection{The Change in Affect between before and after the Reappraisal Task}

The Cronbach's $\alpha$ reliability of the affect scales was high for each type of affect (active positive affect: $\alpha=0.85$, non-active positive affect: $\alpha=0.87$, depression and anxiety: $\alpha=0.89$ ). The mean score of each type of affect at each time is shown in Table 1 and Figures 1-3.

In order to investigate the affect change through the reappraisal task, the change score (Time 3 - Time 2) was calculated for each type of affect and a $t$-test was conducted (Figures 4-6). The result of the $t$-test for active positive affect was significant $(t(105)=2.60, p<0.05)$, and the positive reappraisal group showed more of an increase compared with the putting into perspective group.

Table 1. Means and standard deviations of the mean scores for each type of affect.

\begin{tabular}{ccccccc}
\hline \multirow{2}{*}{ Variable } & \multicolumn{3}{c}{ Positive reappraisal } & \multicolumn{3}{c}{ Putting into perspective } \\
\cline { 2 - 7 } & Time 1 & Time 2 & Time 3 & Time 1 & Time 2 & Time 3 \\
\hline Active positive affect & 11.15 & 8.13 & 9.48 & 11.09 & 8.55 & 8.85 \\
& $(2.72)$ & $(2.32)$ & $(2.42)$ & $(3.38)$ & $(3.71)$ & $(3.75)$ \\
Non-active positive affect & 12.83 & 9.74 & 11.83 & 12.47 & 9.70 & 11.13 \\
& $(3.59)$ & $(2.40)$ & $(3.51)$ & $(3.06)$ & $(3.10)$ & $(3.83)$ \\
Depression and anxiety & 11.31 & 14.06 & 11.50 & 10.81 & 13.17 & 11.98 \\
& $(3.44)$ & $(2.99)$ & $(3.06)$ & $(4.14)$ & $(3.94)$ & $(3.94)$ \\
\hline
\end{tabular}

Note: The upper row shows the means and the lower row shows the standard deviations. 


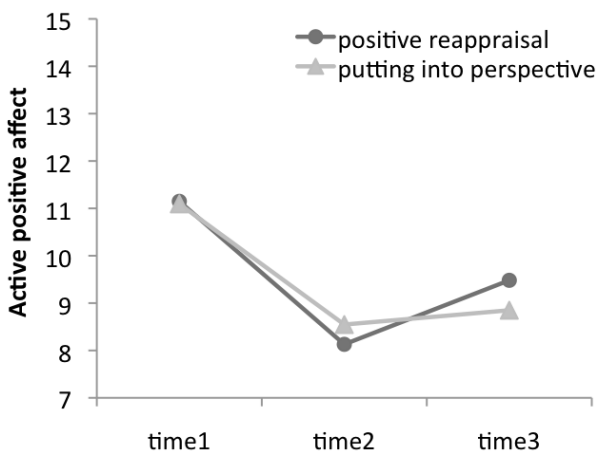

Figure 1. Results of active positive affect.

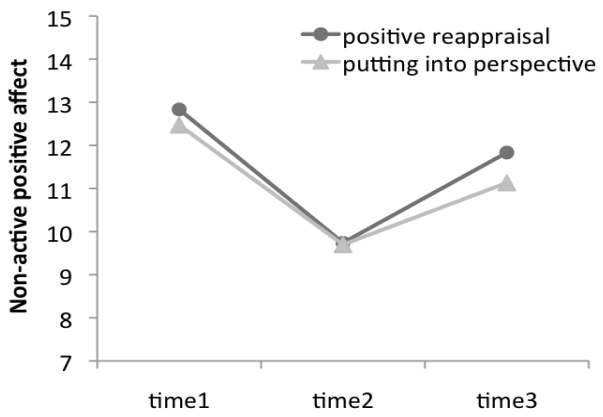

Figure 2. Results of non-active positive affect.

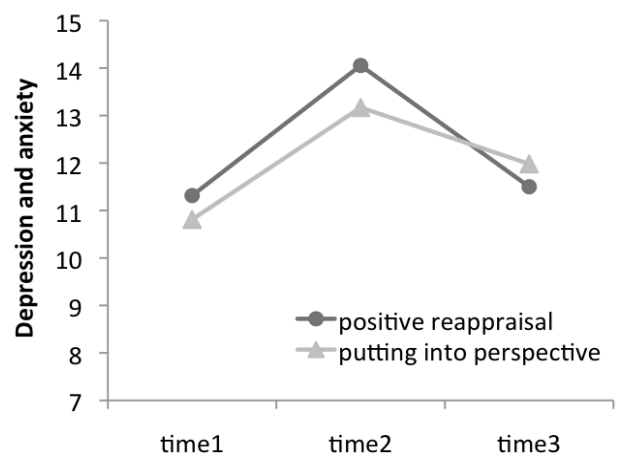

Figure 3. Results of depression and anxiety.

Note: Figures 1-3 show the mean scores for each type of affect at each time point. Time 1 = baseline; Time $2=$ after the stress induction task; Time $3=$ after the reappraisal task.

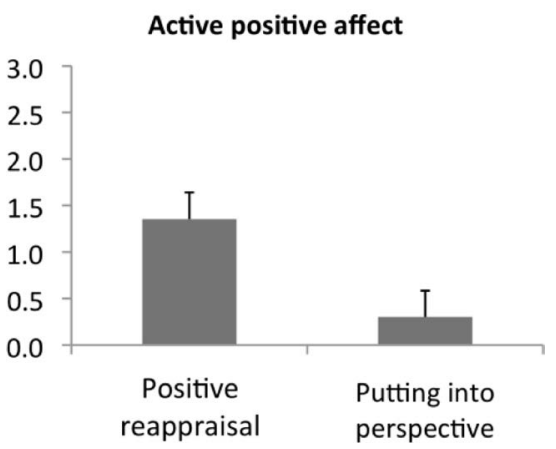

Figure 4. Change in active positive affect. 


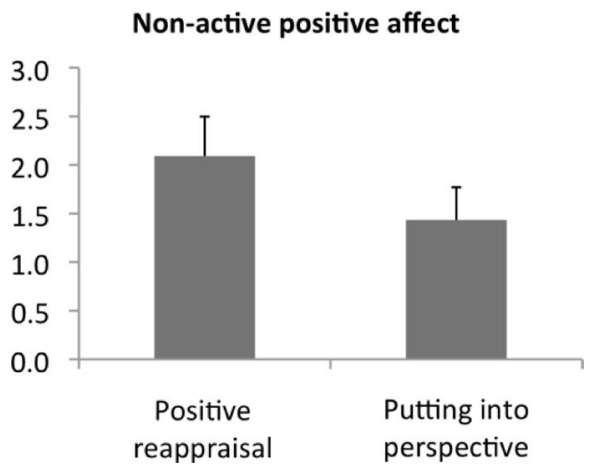

Figure 5. Change in non-active positive affect.

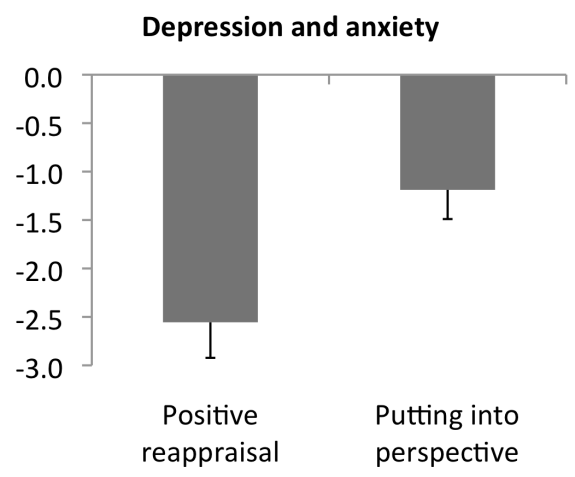

Figure 6. Change in depression and anxiety.

Note: Error bars represent standard errors. Figures 4-6 show the change score for each type of affect in the positive reappraisal and putting into perspective groups.

The result of the $t$-test for non-active positive affect was not significant $(t(105)=$ $1.25)$. The $t$-test result for depression and anxiety was significant $(t(105)=2.87$, $p<0.01$ ), and the positive reappraisal group showed more of a decrease compared with the putting into perspective group.

Finally, the negative affect recovery after the distraction was investigated. A 2 (time) $\times 2$ (group) mixed-model two-way analysis of variance was conducted. The mean score of depression and anxiety at the baseline was $11.31(S D=3.44)$ in the positive reappraisal group and $10.81(S D=4.14)$ in the putting into perspective group. The mean score of depression and anxiety after the distraction task was 9.94 (3.28) in the positive reappraisal group and 9.96 (4.02) in the putting into perspective group. The interaction and main effect of group were not significant $(F(1,105)=1.14, F(1,105)=0.13)$. The main effect of time was significant $(F(1,105)=20.61, p<0.001)$. Both groups showed a significant decrease in depression and anxiety compared to the baseline level.

\section{Discussion}

The purpose of this study was to investigate the effects of two reappraisal subtypes on positive and negative affect. The results of a $t$-test for the change in affect suggested that positive reappraisal was more effective for enhancing active 
positive affect, and reducing depression and anxiety, than the strategy of putting into perspective. Our hypotheses were supported by the results for active positive affect and depression and anxiety. Positive reappraisal is an active type of strategy in terms of the perspective taken, and it focuses on the positive sides of a negative situation, which might be useful for finding the benefits and learning from an event, even though people may not notice these by just thinking about the event.

While we did not have detailed hypotheses about positive affect, the findings also suggest that putting into perspective was as effective for increasing non-active positive affect as positive reappraisal. In previous studies, it was demonstrated that putting into perspective is not necessarily an effective for strategy for emotion regulation. Although putting into perspective is related to positive thoughts, it was proposed that playing down the seriousness of the event is also a less adaptive factor (Schroevers et al., 2007). However, there was no significant difference in non-active positive affect, with both groups exhibiting improved non-active positive affect after the reappraisal task. For a gentle relaxed feeling, putting into perspective is also considered to be effective. Positive cognition types are suggested to include a comparison with a worse event or other people (Hashimoto, 2010). Comparing the negative experience with other undesirable results that the event could have produced is one of the methods that involves thinking alternative thoughts, which might be useful for finding the good fortune in the situation. The findings of this study are meaningful in that this study assessed not only active positive affect but also non-active positive affect.

There are several limitations of this study that future studies will need to address. First, research that includes the other subtypes and considers the context of emotion regulation is necessary. Recent research has also focused on the comparison of other reappraisal subtypes, such as acceptance and distancing (e.g., Kross, Duckworth, Ayduk, Tsukayama, \& Mischel, 2011; Rood et al., 2012). Considering the context of the negative event might also be important for investigating the effects of the subtypes on affect, because the efficacy of a strategy may be different depending on the context (Garnefski \& Kraaij, 2007). Moreover, it is suggested that positive reappraisal might not be effective for strong negative emotion (Sakakibara, 2015). It is necessary to clarify in what context each strategy is more effective. Therefore, it will be required to assess one's evaluation of cognitive appraisal.

Second, although we investigated the short-term effect in this study, it is also necessary to consider the longer term effect of the strategy. Sakakibara \& Kitahara (2016) proposed that there was little long-term effect of putting into perspective, although it could reduce negative emotion in the short term. Because the strategy involves the acceptance that the situation is "good" through comparison with other more negative situations, it does not change the evaluation or meaning of the situation itself. The effects of putting into perspective might be mixed due to the effects of acceptance and resignation. Future research should examine 
the long-term effect in daily life. Third, examining the effects of training that enhances cognitive reappraisal ability will also be fruitful for preventing depression (Troy, Wilhelm, Shallcross, \& Mauss, 2010). We should investigate how people can acquire these skills through an intervention or training. As reappraisal is strongly related to the cognitive-behavioral therapy technique, examining the effect of the subtypes through the experiment in the current study is significant when considering the intervention. It is meaningful to consider a cognitive-behavioral intervention and the prevention of mental health problems based on the effects of the subtypes of reappraisal.

\section{Conclusion}

In conclusion, positive reappraisal was a more effective strategy for emotion regulation than putting into perspective. Using positive reappraisal leads not only to recovery from negative affect but also enhances positive affect. Putting into perspective is also a good strategy for increasing the level of calm affect. However, there might be situations in which using positive reappraisal effectively is difficult. Although this study focused on only two strategies, investigating the effects of reappraisal on emotion regulation by including other subtypes might be useful for employing an adaptive strategy corresponding to the situation or for developing a more effective intervention program of reappraisal. The subtypes' contents were different in each previous research study; therefore, it is desirable to examine what kind of reappraisal is effective when considering the context of emotion regulation and what factors influence the efficacy of the subtypes. Since reappraisal is one of the most representative emotion regulation strategies, it is expected that examining the effects of the subtypes of reappraisal on affect in detail will contribute greatly to emotion regulation research and practice in daily life.

\section{Acknowledgements}

This work was supported by JSPS KAKENHI Grant Number JP16K04346.

\section{References}

Garnefski, N., \& Kraaij, V. (2007). The Cognitive Emotion Regulation Questionnaire: Psychometric Features and Prospective Relationships with Depression and Anxiety in Adults. European Journal of Psychological Assessment, 23, 141-149. http://hdl.handle.net/1887/14248

Garnefski, N., Kraaij, V., \& Spinhoven, P. (2001). Negative Life Events, Cognitive Emotion Regulation and Emotional Problems. Personality and Individual Differences, 30, 1311-1327.

Gross, J. J. (1998a). The Emerging Field of Emotion Regulation: An Integrative Review. Review of General Psychology, 2, 271-299. https://doi.org/10.1037/1089-2680.2.3.271

Gross, J. J. (1998b). Antecedent- and Response-Focused Emotion Regulation: Divergent Consequences for Experience, Expression, and Physiology. Journal of Personality and Social Psychology, 74, 224-237. http://dx.doi.org/10.1037/0022-3514.74.1.224 
Hashimoto, K. (2010). Positive Cognitions in Various Stressful Events: That Have Different Severities in Different Fields. Kyoto University Research Studies in Education, 56, 425-437. http://hdl.handle.net/2433/108463

Hofmann, S. G., Heering, S., Sawyer, A. T., \& Asnaani, A. (2009). How to Handle Anxiety: The Effects of Reappraisal, Acceptance, and Suppression Strategies on Anxious Arousal. Behaviour Research and Therapy, 47, 389-394.

Jermann, F., Van der Linden, M., d’ Acremont, M., \& Zermatten, A. (2006). Cognitive Emotion Regulation Questionnaire (CERQ): Confirmatory Factor Analysis and Psychometric Properties of the French Translation. European Journal of Psychological Assessment, 22, 126-131. https://doi.org/10.1027/1015-5759.22.2.126

Kross, E., Duckworth, A., Ayduk, O., Tsukayama, E., \& Mischel, W. (2011). The Effect of Self-Distancing on Adaptive versus Maladaptive Self-Reflection in Children. Emotion, 11, 1032-1039. https://doi.org/10.1037/a0021787

McRae, K., Ciesielski, B., \& Gross, J. J. (2012). Unpacking Cognitive Reappraisal: Goals, Tactics, and Outcomes. Emotion, 12, 250-255. https://doi.org/10.1037/a0026351

Nolen-Hoeksema, S., Wisco, B. E., \& Lyubomirsky, S. (2008). Rethinking Rumination. Perspectives on Psychological Science, 3, 400-424. https://doi.org/10.1111/j.1745-6924.2008.00088.x

Rood, L., Roelofs, J., Bögels, S. M., \& Arntz, A. (2012). The Effects of Experimentally Induced Rumination, Positive Reappraisal, Acceptance, and Distancing When Thinking about a Stressful Event on Affect States in Adolescents. Journal of Abnormal Child Psychology, 40, 73-84. https://doi.org/10.1007/s10802-011-9544-0

Sakakibara, R. (2014). Emotion Regulation Effects of Reappraisal and Its Influences on Psychological Well-Being: Review of Research Trends and Discussions from the Perspective of the Subtypes of Reappraisal. Japanese Journal of Research on Emotions, 22, 40-49.

Sakakibara, R. (2015). The Usage Tendency of and the Relation with Psychological Health of Cognitive Emotion Regulation: Development of Japanese-Version Cognitive Emotion Regulation Questionnaire and Focus on the Effects of Negative Emotional Intensity. Japanese Journal of Research on Emotions, 23, 46-58. https://doi.org/10.4092/jsre.23.46

Sakakibara, R., \& Kitahara, M. (2016). The Relationship between Cognitive Emotion Regulation Questionnaire (CERQ) and Depression, Anxiety: Meta-Analysis. The Japanese Journal of Psychology, 87, 179-185. https://doi.org/10.4992/jjpsy.87.15302

Schroevers, M., Kraaij, V., \& Garnefski, N. (2007). Goal Disturbance, Cognitive Coping Strategies, and Psychological Adjustment to Different Types of Stressful Life Event. Personality and Individual Differences, 43, 413-423. https://doi.org/10.1016/j.paid.2006.12.009

Terasaki, M., Kishimoto, Y., \& Koga, A. (1992). Construction of a Multiple Mood Scale. The Japanese Journal of Psychology, 62, 350-356. https://doi.org/10.4992/jipsy.62.350

Troy, A. S., Wilhelm, F. H., Shallcross, A. J., \& Mauss, I. B. (2010). Seeing the Silver Lining: Cognitive Reappraisal Ability Moderates the Relationship between Stress and Depressive Symptoms. Emotion, 10, 783-795. https://doi.org/10.1037/a0020262

Webb, T. L., Miles, E., \& Sheeran, P. (2012). Dealing with Feeling: A Meta-Analysis of the Effectiveness of Strategies Derived from the Process Model of Emotion Regulation. Psychological Bulletin, 138, 775-808. https://doi.org/10.1037/a0027600 